\title{
The Computer Simulation of Radiation Pattern for Cylindrical Conformal Microstrip Antenna
}

\author{
Ruying Sun \\ School of Informatics, Linyi Normal University, Linyi 276005, China \\ E-mail: srysd@163.com
}

\begin{abstract}
FEKO is a 3D simulation tool for the analysis of electromagnetic fields. The simulation using FEKO is based on the well-known method of moments (MoM) for the Maxwell equations. FEKO can solve the problem of any structural type with a very comprehensive MoM code or tailored code. First, a radiation pattern for conformal microstrip antenna on the surface of cylinder is calculated using FEKO. In addition, the impact of conformal carrier on the radiations of microstrip antennas in different positions is simulated by FEKO. The simulation results accord with the true characteristics of conformal microstrip antennas.
\end{abstract}

Keywords: Computer simulation, Conformal antenna, Radiation pattern, FEKO

\section{Introduction}

Conformal antenna technology is closely linked the development of microwave integrated technology and the development of space information technology. A variety of advanced aircraft and transport, such as aircrafts, missiles, satellites, ships and vehicles, more and more are requested to make their radar antennas to be conformal (Kin Lu Wong, 1999, chapter1).

Conformal antennas are mainly the following advantages:

(1) Conformal antennas have little effect of carrier's own aerodynamic properties due to its conformal surface.

(2) Conformal antennas can be used to simplify the antenna installation under the conditions of assuring the performance of the antenna.

(3) Conformal antennas can eliminate or reduce the error caused by radome. The radome does not need to install; even if the radome is installed, as the microstrip antenna and its close proximity, distortion of the antenna is greatly reduced (B. P. Kumar, 2005, 623 ).

Because of the advantages of conformal antennas, it is very popular in the different flight aircrafts (Kene J.Allard, 2003, 1055 ).

\section{Conformal Antenna Radiation Pattern}

In the paper, the research of conformal antenna units is based on the relatively small curvature radius of cylinder (in small electrical dimension).

The specific coordinate and model are shown in Figure 1. The center of rectangular microstrip antenna on the cylinder at the point (according to cylindrical coordinate system) $\left(a, 0^{\circ}, 0\right)$, in which the radius of cylinder is a, the length of cylinder is $4 \lambda$. The length of rectangular patch is $2 L$ and width is $2 \varphi_{0} a$. The relative dielectric constant for dielectric is $\varepsilon_{\mathrm{r}}, \varepsilon_{r}=2.33$, the thickness of the substrate is $h, h=0.05 \lambda_{g}$, where $\lambda_{g}$ is the medium wavelength, $\lambda_{g}=\lambda / \sqrt{\varepsilon_{r}}$. In unfolded diagram of the conformal microstrip antenna (Figure 2), the length of the direction $z$ is the cylinder height, width is the half of cylinder circumference, the size of the patch is the same as which is in Figure 1. The microstrip antenna is fed using probe; the probe is not in the patch center, but at the point which is $0.125 \lambda_{g}$ away from the center along the direction $\varphi$.

For the above structure, the simulation of conformal microstrip antenna with FEKO 4.0 is carried out. The structure used in simulating is shown in Figure 3. The simulation results are shown in figure 4 when the cylinder has different radius.

Several conclusions can be found by observing Figure 4, because of the location of feed led to an asymmetric pattern at 
plane $\mathrm{H}$, and merely no impact on plane $\mathrm{E}$. When the other conditions remain unchanged, with the larger radius of the cylinder being of, the pattern has smaller back beam at plane E, which meet to the truth the back beam of the microstrip antenna almost disappeared when its substrate tends to infinity. When the radius of the cylinder increased, back beam of the antenna will also decrease accordingly. In general, the results of this simulation show a truth on radiation characteristics of a conformal microstrip antenna.

\section{The Impact of Carrier for Conformal Radiation patterns}

In order to study the impact of carrier for radiation patterns, a case in Figure 5 is carried out. There are 8 elements of an array on the surface of a cylinder, each of which is calculated separately. When calculating the current element, other elements are regarded as metal scatterers. The location of each element are shown in Figure 5, the distance of adjacent element is $d=0.5 \lambda$, the geometric dimensions of the cylinder and the array element are the same as which in Figure 2. The results of the case are shown in Figure 6.

From the figure we can see the analytical results: element patterns' symmetrical relationship can rooted from the geometry of symmetric relations; when the array element in the location of the end of the cylinder, the pattern of the antenna has more dramatic the impact. Major changes in patterns as follows: from the end draws nearer, the greater the pattern backbeam; the patterns' distortion occur at the last element.

Two elements' patterns at both ends occur sharp changes in direction, mainly because the two ends of cylinder geometry changes in the structure of the surface caused a sudden sharp changes in surface currents, together with two end faces is the metal surface, the induced current the surface is generally greater than the strength of the surface current on media surface (J.A. Ferreira, 1997, 1088 ).

\section{Conclusion}

In this paper, the radiation patterns are simulated using FEKO 4.0. One is to simulate the radiation pattern of a conformal microstrip antenna on the surface of cylinder; the other simulation talked about the impact of conformal carrier on the radiation patterns of microstrip antennas in different positions. The results show that FEKO 4.0 has good precision and high efficiency in simulation of conformal microstrip antennas. This study has great significance to talking about conformal array antennas.

\section{References}

B. P. Kumar. (2005). Generalized Analytical Technique for the Synthesis of Unequally Spaced Arrays With Linear, Planar, Cylindrical or Spherical Geometry. IEEE Transactions on antennas and Propagation, VOL.53, NO.1, 621-634.

J.A. Ferreira and F. Ares. (1997). Pattern synthesis of conformal arrays by the simulated annealing technique. IEE Electronics Letters, Vol. 33 No.14, 1187-1189.

Kene J.Allard, Donglas H.Werner and Pingjuan L.Werner. (2003). Radiation Pattern Synthesis for Arrays of Conformal Antennas Mounted on Arbitrarily-Shaped Three-Dimensional Platforms Using Genetic Algorithms. IEEE transactions on antennas and propagation, Vol.51, No.5, 1054-1062.

Kin Lu Wong.( 1999). Design of Nonplanar Microstrip Antennas and Transmission Lines, John Weily \& Sons, Inc, $1-100$.

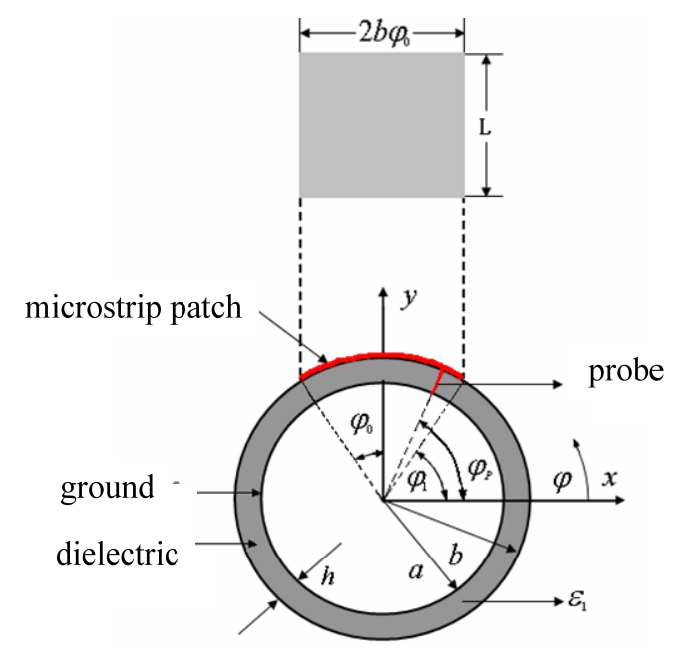

Figure 1. Cross-section diagram of the conformal microstrip antenna 


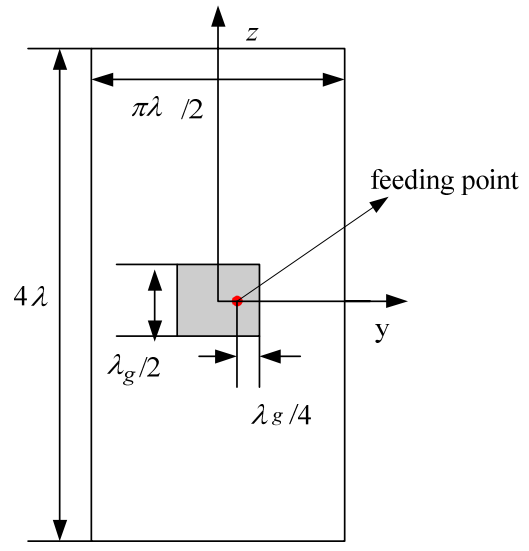

Figure 2. Unfolded diagram of the conformal microstrip antenna

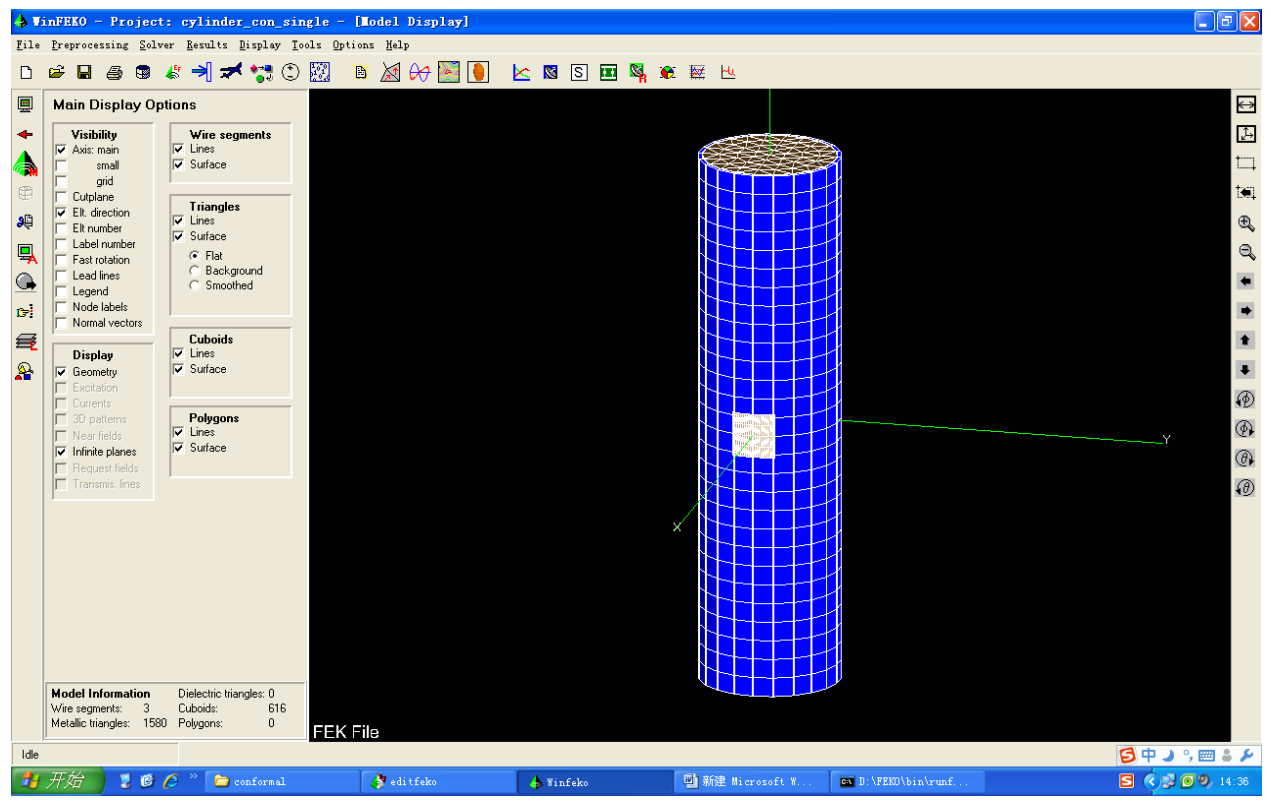

Figure 3. The structure in WinFEKO surroundings of KEKO 4.0.

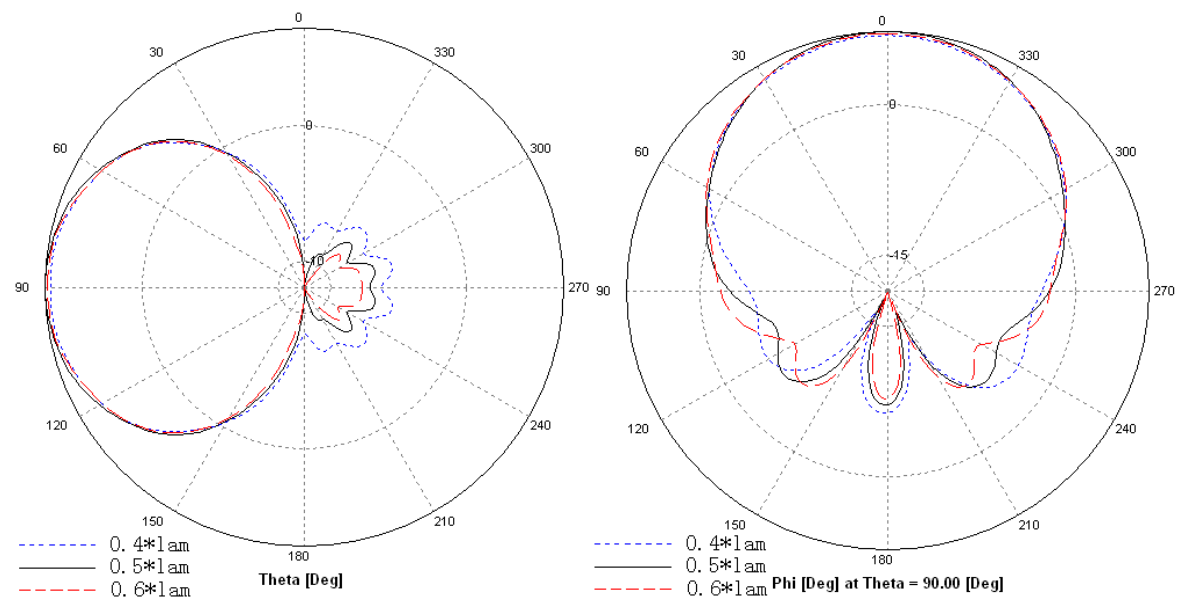

(a) Plane E

(b) Plane $\mathrm{H}$

Figure 4. The radiation patterns at plane $\mathrm{E}$ and plane $\mathrm{H}$ for conformal microstrip antenna when the cylinder of different radius. Three numerical outcomes are given, they are shown with three curves when $a=0.4 \lambda, a=0.5 \lambda$ and $a=0.6 \lambda$. 


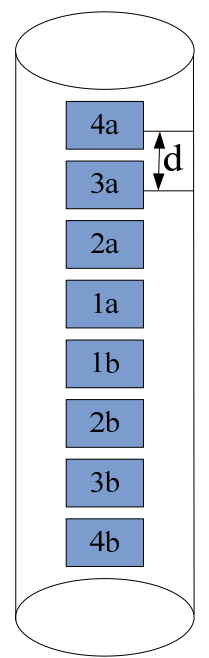

Figure 5. 8 array elements placed on finite length cylinder. The elements above the center of the cylinder have the naming suffix "a", and the elements below the center of the cylinder have the naming suffix "b".

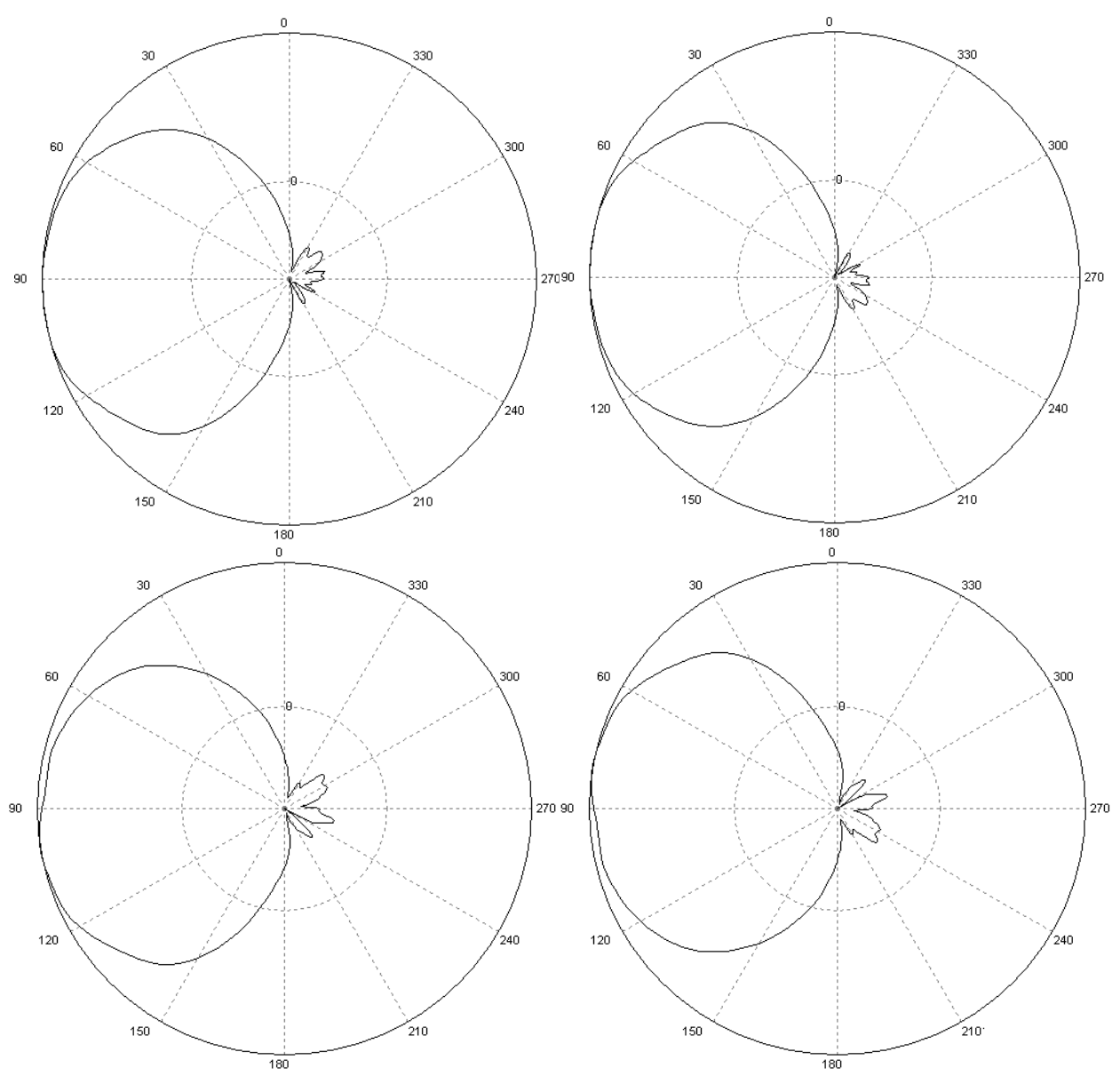




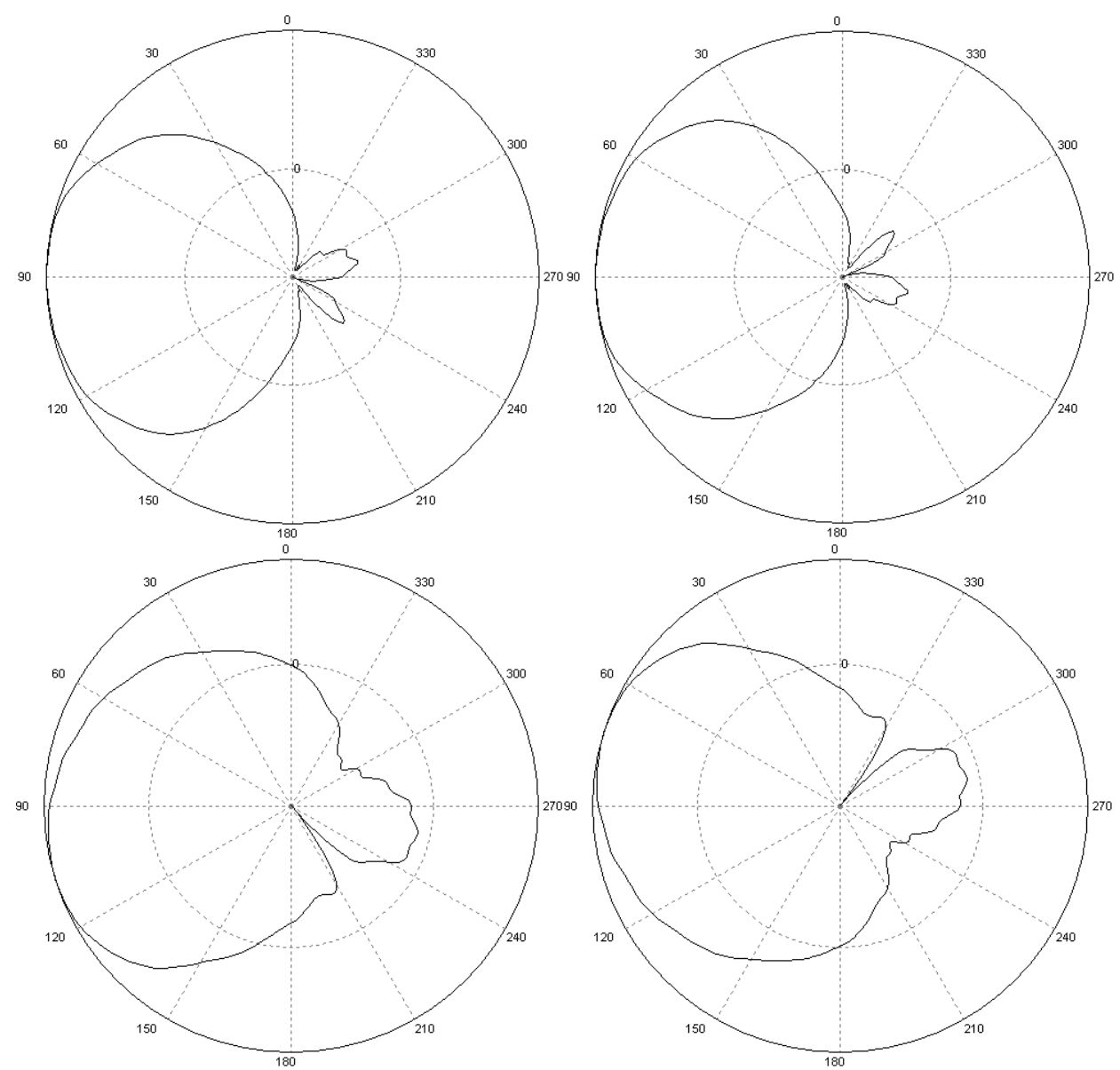

Figure 6 . The patterns for 8 array elements. By line number, the pattern is respectively for $1 \mathrm{a}, 1 \mathrm{~b}, 2 \mathrm{a}, 2 \mathrm{~b}, 3 \mathrm{a}, 3 \mathrm{~b}, 4 \mathrm{a}$ and $4 \mathrm{~b}$ element. 